\title{
Addition of coconut oil to the diet based on maize dried distilled grains with solubles (DDGS) alters miR-122a expression in the pig liver
}

\author{
M. Oczkowicz ${ }^{1,3}$, K. Pawlina ${ }^{2}$, M. Bugno-Poniewierska ${ }^{2}$ and M. Świątkiewicz ${ }^{1}$ \\ National Research Institute of Animal Production \\ ${ }^{1}$ Department of Animal Genetics and Breeding, ${ }^{2}$ Laboratory of Genomics \\ Krakowska 1, $32-083$ Balice, Poland
}

KEY WORDS: microRNA, nutrigenomics, dietary fat, miR-122a, pigs

Received: $\quad 4$ April 2017

Revised: 3 October 2017

Accepted: 10 December 2017

${ }^{3}$ Corresponding author:

e-mail: maria.oczkowicz@izoo.krakow.pl

\begin{abstract}
The aim of the study was to analyse the expression of several microRNAs (miRNA) in the liver of pigs fed different diets. Twenty-four fatteners (12 gilts and 12 barrows) originating from (Polish Landrace $\times$ White Large Polish) sows mated with (Duroc $\times$ Pietrain) boars were divided into 4 dietary groups, with 6 pigs in each group ( 3 gilts and 3 barrows). The fattening experiment lasted from about $60 \mathrm{~kg}$ to $118 \mathrm{~kg}$ of body weight. The animals were fed diets that differ with the presence of maize dried distilled grains with solubles (DDGS; groups II, III, IV - 20\%) and the type of used fat (rapeseed oil - groups I and II, beef tallow - group III, coconut oil - group IV). A qPCR analysis to assess miR-148a-3p, miR-122a, miR-26a, miR-103, let-7a, miR-92a, miR-335 and miR-16a expressions was performed. In the GeNorm software analysis it was shown that the most stably expressed miRNAs were miR-26a, miR-16a and miR-148a-3p (M values: $0.51-0.52$ ). Only miR-122a expression was different $(P<0.03)$. The miR-122a level was statistically lower in the liver of pigs from group IV (DGS+coconut oil). The results suggest that adding coconut oil to feedstuff based on maize DDGS changes the expression of miR-122a, which is an important regulator of many genes engaged in lipid metabolism.
\end{abstract}

\section{Introduction}

Over the last decade, increased attention has been put on the association between nutrients and genome, resulting in the rise of two new branches of knowledge - nutrigenetics and nutrigenomics. The main goal of nutrigenetics is to establish the effect of genetic variations on the interaction between diet and disease or on nutrient requirements, while nutrigenomics is focused on the effect of nutrients on the genome, proteome and metabolome (Müller and Kersten, 2003). There is a growing evidence of such effects as well as of the strong interaction between genotype and nutrients in modulating animal phenotypes (Jiménez-Chillarón et al., 2012).

Nowadays, it is very important to feed farm animals with appropriate feedstuff as this guarantees high-quality animal products and breeding profitability. Recently, dried distilled grains with solubles (DDGS) have become very popular in pig feeding as DDGS contain large amounts of protein and are more reasonably priced than other feedstuffs. Furthermore, DDGS are a good source of unsaturated fatty acids, which are preferable for the health of the animal but 
negatively affect the technological parameters of the fat. The fat in animals fed large amounts of unsaturated fatty acids shows decreased firmness and is more susceptible to oxidation. To counter this effect, feeds containing large quantities of saturated fatty acids from different sources, like coconut oil or beef tallow, are added to diets.

MicroRNAs (miRNAs) are small, non-coding RNAs ( $21-23 \mathrm{nt})$ that have the ability to influence gene expression by binding to the 3'UTR region of mRNA. MicroRNA coding genes can occur in intergenic regions as well as in the exons and introns of both coding and non-coding genes. Processing the miRNA transcript (pri-miRNA) with Drosha enzyme generates precursory miRNA (pre-miRNA) (Lee et al., 2003). Mature miRNA is then excised by Dicer ribonuclease and incorporated into the protein RNA-induced silencing complex (RISC). The miRNA-RISC complex binds to the 3'UTR of mRNA, causing translational repression and degradation or sometimes upregulation of expression (Vasudevan et al., 2007).

MicroRNAs are believed to play a significant role in a wide variety of biological processes, such as cardiovascular diseases, oncogenesis, development, apoptosis and cholesterol metabolism (Moore et al., 2010). New aspects of miRNAs functioning are still being discovered, and an increasing number of miRNA-focused analyses are being conducted (Tavazoie et al., 2008). Recently, miRNAs connected with nutrient metabolism and diet have been under scrutiny (Saini et al., 2010). The expression of several miRNAs, including the miR-21 family, miR-200 family, miR-17 cluster and let-7 family, is affected by dietary factors. The levels of these miRNAs may be altered by polyunsaturated fatty acids (PUFA), proteins, glucose, fibre, folate and others (Palmer et al., 2014). Moreover, nutrients and other bioactive food components (BFC), such as (n-3) fatty acids or selenium, are thought to have an influence on cancer occurrence (Ross and Davis, 2011) because of their ability to modulate miRNA expression. However many miRNA features, such as mRNA targets of many mammalian miRNAs, specific functions and mechanisms have still not been characterized thoroughly and need to be further investigated (Thomson et al., 2011).

Due to the application of cutting-edge technologies in recent years, e.g., next-generation sequencing or microarrays, significant improvement has been made in the field of miRNA identification in different species. To date, according to miRBase 21.0 (Griffiths-Jones et al., 2006, 2008), 2578 human miRNAs have been deposited, whereas almost 5 years ago, this number exceeded only 700 (miRBase 14.0). In other mammals, especially livestock species, coverage of the miRNAome is still sparse, despite obvious improvement over the years. Pigs with 77 miRNAs in miRBase 14.0 vs 326 in miRBase 20.0 are a good example of such animals. Also, due to the fact that they are phylogenetically relative to humans they play important role in livestock production as well as in biomedical research. So, it is essential to gain an insight into mechanisms that modulate gene expression and influence organism functioning in pigs (Sartor, 2005), especially in the liver as a highly specialized organ that participates in a wide variety of metabolic processes and is crucial to vital functions.

Recently, we performed a whole transcriptome study on gene expression in the pig liver when different source of fats were added to diets containing DDGS (Oczkowicz et al., 2016). A set of differentially expressed genes (DEG) in samples of the liver from pigs fed rapeseed oil, coconut oil and beef tallow as a source of fat in the diet was identified. Expression differences were confirmed by qPCR for five DEG (APOA4, ACSL5, CYP2C49, CYP2B22 and GSTO1). To identify the possible regulatory mechanism of these genes, miRNAs specific to the liver of pigs were investigated (Pawlina et al., 2015). By employing next-generation sequencing, 206 distinct miRNAs were identified. Of these, 138 are deposited in the miRBase and 68 are potentially novel miRNAs. In the present study, the focus is placed on the miRNAs that were highly expressed in the liver (miR-122a, miR-148a-3p, let-7a, miR-103 and miR-26a) in accordance with previous studies (Gamazon et al., 2013). So, the aim of the study was to investigate which selected miRNAs specific to the porcine liver would be affected by the diet and which would be stable. To increase the probability of identifying nutrient-regulated miRNAs, the attention was paid to putative miRNAs that may affect expression of DEG which was previously identified in our whole transcriptome study.

\section{Material and methods}

\section{Animals}

Twenty-four fatteners (12 gilts and 12 barrows) originating from (Polish Landrace $\times$ White Large Polish) sows mated with (Duroc $\times$ Pietrain) boars were divided into 4 dietary groups, with 6 pigs in each group (3 gilts and 3 barrows). Animals were 
kept in individual straw-bedded pens with free access to water. The fattening experiment lasted from about $60 \mathrm{~kg}$ to $118 \mathrm{~kg}$ of body weight (BW). All procedures relating to the use of live animals included in the experiment were in agreement with the II Local Ethics Committee for Experiments with Animals (Cracow, Poland, Resolution No. 912).

\section{Diets and treatment}

The energy and protein concentrations in all diets (metabolizable energy $\sim 13.4 \mathrm{MJ}$, crude protein $\sim 165$ g, Lys $\sim 9.1 \mathrm{~g}$, Met+Cys $\sim 5.9 \mathrm{~g}$, Thr $\sim 5.8$ g, Trp $\sim 1.9$ g, crude fat $\sim 49 \mathrm{~g} \cdot \mathrm{kg}^{-1}$ ) were similar and covered the nutritional requirements for pigs. Mash diets included barley, wheat, maize, wheat bran, soyabean meal, vitamin-mineral additives and crystalline amino acids but differed in the addition of maize DDGS (groups II, III, IV - 20\%) and the type of used dietary fat (rapeseed oil - groups I and II, beef tallow - group III, coconut oil - group IV). Feed mixtures differed significantly in their fatty acid profiles and saturated to unsaturated fatty acids (SFA:UFA) ratios (Table 1). The feed mixtures for groups III and IV were characterised by higher amount of SFA and lower iodine value of fat as compared with groups I and II. All pigs were fed restricted feed amounts twice a day according to body weight: from $2.8 \mathrm{~kg} \cdot \mathrm{d}^{-1}$ at $61-70 \mathrm{~kg} \mathrm{BW}$ and $3.0 \mathrm{~kg} \cdot \mathrm{d}^{-1}$ at $71-80 \mathrm{~kg}$ BW to $3.2 \mathrm{~kg} \cdot \mathrm{d}^{-1}$ at $80 \mathrm{~kg}$ of BW. Dietary experiment lasted 59-61 days.

\section{Sample collection}

At the end of the fattening, all pigs were slaughtered. Stunning with high-voltage electric tongs (voltage $240-400 \mathrm{~V}$ ) was followed by exsanguination. Immediately after slaughter, the same section of liver samples were collected from 6 animals in each group for miRNA expression analysis. Immediately after separation, samples $\left(\sim 1 \mathrm{~cm}^{2}\right)$ were frozen in liquid nitrogen and kept in a freezer $\left(-85^{\circ} \mathrm{C}\right)$.

\section{RNA isolation}

Total RNA was isolated from $\sim 10 \mathrm{mg}$ of liver, using Directzol RNA kit (Zymo Research, Irvine, CA, USA according to the manufacturer's protocol. The tissue was homogenized in Trireagent (Thermofisher, Waltham, MA, USA) using Bullet blender 24 (Next Advance, New York, NY, USA). The RNA integrity was checked by electrophoresis on $2 \%$ agarose gel.

\section{Reverse transcription and qPCR}

Reverse transcription was performed using $5 \mathrm{ng}$ of total RNA, miRNA specific primers and TaqMan ${ }^{\mathbb{B}}$ MicroRNA Reverse Transcription Kit (Thermofisher,
Waltham, MA, USA), according to the manufacturers protocol. qPCR was conducted on an Eco instrument (Illumina, San Diego, CA, USA) using TaqMan ${ }^{\circledR}$ MicroRNA Assays (Thermofisher, Waltham, MA, USA) as follows: $000470-$ miR-148a-3p, $000445-$ miR122a, 000405 - miR-26a, 000439 - miR-103, 000377 - let-7a, and TaqMan ${ }^{\circledR}$ Universal MasterMix II with UNG (Thermofisher, Waltham, MA, USA), according to the manufacturer's protocol.

\section{Statistical analysis and relative quantification calculation}

The miRNA with the most stable expression was selected as endogenous control with use of GeNorm software analysis (https://genorm.cmgg.be) (Vandesompele et al., 2002). The relative quantity of analysed miRNAs was calculated using the equation described by Pfaffl (2001). The miRNA expression comparison across different feeding groups was performed using the GLM procedure (SAS Enterprise guide 7.1, SAS Inc., Cary, NC, USA), with relative quantity (RQ) and sex as fixed effects. The data were normally distributed (tested with Kolmogorov-Smirnov test).

\section{Results}

\section{Animal performance}

Various types of dietary fats significantly changed the fatty acids profiles and SFA:UFA ratios of the feed mixtures (Table 1). The diet of group II was characterized by a higher UFA content, especially c18:2 linoleic acid. Also, in groups III and IV, a higher SFA content and lower iodine value were observed. In the group IV a SFA:UFA ratio above 1 was found, and more than half of all SFA were c9-c12 fatty acids.

In the present experiment, dietary treatment did not significantly impact weight gain or feed utilization in pigs (Świątkiewicz et al., 2016). The average daily weight gain during the trial $(60-118 \mathrm{~kg})$ in all groups ranged from 958 to $991 \mathrm{~g}$, and feed utilization ranged from 3.2 to $3.0 \mathrm{~kg}$. Similarly, meat content in carcasses $(\sim 55 \%)$, weight of primal cuts $(\sim 26 \mathrm{~kg})$, average back fat thickness $(\sim 2.2 \mathrm{~cm})$ and liver weight $(\sim 1.8 \mathrm{~kg})$ remained unchanged. A significant effect of dietary treatment on the fatty acid profiles and SFA:UFA ratios of subcutaneous adipose tissue in back fat was observed, described and discussed by Świątkiewicz et al. (2016). Subcutaneous adipose tissue in the back fat of animals receiving maize DDGS and rapeseed oil (group II) contained significantly higher amount of 
Table 1. Composition and fatty acid content $\left(\mathrm{g} \cdot 100 \mathrm{~g}^{-1}\right.$ of estimated fatty acids) of diets

\begin{tabular}{|c|c|c|c|c|}
\hline Indices & $\begin{array}{l}\text { Group I (control) } \\
\text { diet with rapeseed oil }\end{array}$ & $\begin{array}{l}\text { Group II } \\
\text { diet with rapeseed oil + } \\
\text { maize DDGS }\end{array}$ & $\begin{array}{l}\text { Group III } \\
\text { diet with beef tallow } \\
+ \text { maize DDGS }\end{array}$ & $\begin{array}{l}\text { Group IV } \\
\text { diet with coconut fat } \\
+ \text { maize DDGS }\end{array}$ \\
\hline \multicolumn{5}{|l|}{ Diet composition, $\mathrm{g} \cdot 100 \mathrm{~g}^{-1}$} \\
\hline barley & 43.57 & 41.61 & 41.61 & 41.61 \\
\hline wheat & 25.00 & 25.00 & 25.00 & 25.00 \\
\hline maize & 5.00 & - & - & - \\
\hline soyabean meal & 16.00 & 8.00 & 8.00 & 8.00 \\
\hline wheat bran & 5.00 & - & - & - \\
\hline DDGS & - & 20.00 & 20.00 & 20.00 \\
\hline rapeseed oil & 3.00 & 3.00 & - & - \\
\hline beef tallow & - & - & 3.00 & - \\
\hline coconut oil & - & - & - & 3.00 \\
\hline vitamin-mineral premix & 0.50 & 0.50 & 0.50 & 0.50 \\
\hline salt & 0.22 & 0.21 & 0.21 & 0.21 \\
\hline fodder phosphate & 0.17 & 0.10 & 0.10 & 0.10 \\
\hline fodder chalk & 1.30 & 1.20 & 1.20 & 1.20 \\
\hline L-Lysine & 0.18 & 0.33 & 0.33 & 0.33 \\
\hline DL-Methionine & 0.03 & - & - & - \\
\hline L-Threonine & 0.03 & 0.02 & 0.02 & 0.02 \\
\hline L-Tryptophan & - & 0.03 & 0.03 & 0.03 \\
\hline \multicolumn{5}{|c|}{ Fatty acids composition, $\mathrm{g} \cdot 100 \mathrm{~g}^{-1}$} \\
\hline c8 & 0.13 & 0.07 & 0.13 & 3.81 \\
\hline $\mathrm{c} 10$ & - & - & 0.19 & 3.53 \\
\hline$c 12$ & 0.50 & 0.26 & 0.76 & 25.1 \\
\hline c14 & 0.25 & 0.25 & 0.95 & 6.85 \\
\hline $\mathrm{c} 16$ & 12.4 & 11.0 & 19.8 & 11.6 \\
\hline$c 16: 1$ & 0.20 & 0.18 & 0.86 & 0.18 \\
\hline$c 18$ & 2.35 & 2.56 & 8.96 & 2.50 \\
\hline c18:1 & 43.0 & 35.5 & 31.2 & 15.2 \\
\hline c18:2 & 30.3 & 41.1 & 31.9 & 27.7 \\
\hline$c 18: 3$ & 5.27 & 5.28 & 2.72 & 1.97 \\
\hline$\gamma c 18: 3$ & 0.06 & - & 0.02 & - \\
\hline $\mathrm{c} 20$ & 0.96 & 0.81 & 0.53 & 0.36 \\
\hline c20:4 & 0.04 & 0.03 & 0.09 & 0.02 \\
\hline c20:5 (EPA) & 0.14 & 0.10 & 0.05 & 0.05 \\
\hline c22 & 0.71 & 0.50 & 0.22 & 0.19 \\
\hline $\mathrm{c} 22: 1$ & 1.22 & 0.77 & 0.43 & 0.33 \\
\hline $\mathrm{c} 22: 6(\mathrm{DHA})$ & 0.62 & 0.43 & 0.21 & 0.15 \\
\hline SFA & 17.35 & 15.5 & 31.6 & 53.9 \\
\hline UFA & 80.9 & 83.4 & 67.5 & 45.6 \\
\hline MUFA & 44.4 & 36.4 & 32.4 & 15.7 \\
\hline PUFA n-6 & 30.4 & 41.1 & 32.1 & 27.7 \\
\hline PUFA n-3 & 6.04 & 5.80 & 2.98 & 2.16 \\
\hline PUFA & 36.4 & 46.9 & 35.1 & 29.9 \\
\hline SFA:UFA ratio & 0.21 & 0.19 & 0.47 & 1.18 \\
\hline iodine value & 104.5 & 116.2 & 90.5 & 66.6 \\
\hline
\end{tabular}

maize DDGS - maize dried distilled grain with solubles; SFA - saturated fatty acids; UFA - unsaturated fatty acids; MUFA - monounsaturated fatty acids; PUFA - polyunsaturated fatty acids

UFA and lower SFA content (lower SFA:UFA ratio) in comparison to remaining groups $(P<0.001)$. The back fat of group II was characterized by the highest content of c18:2 linoleic acid, which is negatively related to fat hardness, an important attribute in the processing of meat products. In addition, this finding indicates higher susceptibility to the lipid oxidative process.

\section{Selection of miRNAs by in silico MirTarBase analysis}

The MirTarBase (http://mirtarbase.mbc.nctu. edu.tw/php/index.php) - a database that contains information regarding experimentally proven miRNA-gene interactions - was scanned to find putative miRNAs that may regulate expression of the differentially expressed genes that we pre- 
viously identified in a RNA-Seq experiment (Oczkowicz et al., 2016; Świątkiewicz et al., 2016). The 61 distinct miRNAs were identified. Of these, miRNAs that are highly expressed in the liver (miR-103, miR-16a, miR-122a, miR-148a-3p, miR-26a, miR92a) (Pawlina et al., 2015) were selected. Furthermore, the miR-335 was included in the study despite the fact that in the previous study (Pawlina et al., 2015) we did not detect it in the liver miRNAome by NGS (Next Generation Sequencing) but as many as seven DEG were possible targets for this miRNA. The let-7a was also included in the experiment because it has been shown to be a good endogenous control in the porcine liver (Timoneda et al., 2012).

\section{Identification of the most stable liver miRNAs after diet treatment}

qPCR analysis confirmed that all of the analysed miRNAs were highly expressed in the liver, except miR-335. For all miRNAs but one (miR-335), $\mathrm{C}_{\mathrm{t}}$ values were below 30 . The most abundant miRNA was miR-26a (mean $C_{t}=21.6$ ), whereas miR-335 was weakly expressed in the liver (mean $\mathrm{C}_{t}=31.7$ ). Since little is known about the appropriate endogenous control for qPCR analysis of miRNA after diet treatment, the stability of all eight miRNAs using the GeNorm software was analysed. According to this analysis, all miRNAs had relatively low (between $0.5-1.78) \mathrm{M}$ values - a parameter determining stability. Nevertheless, the most stable were miR-26a, miR-16 and miR-148a-3p (M values: 0.51-0.52). The least stable was miR-335 ( $M=1.78)$, while let-7a, miR-103 and miR-122a presented intermediate values ( $\mathrm{M}=0.8220, \mathrm{M}=0.724$ and $\mathrm{M}=0.598$, respectively) (Figure 1).

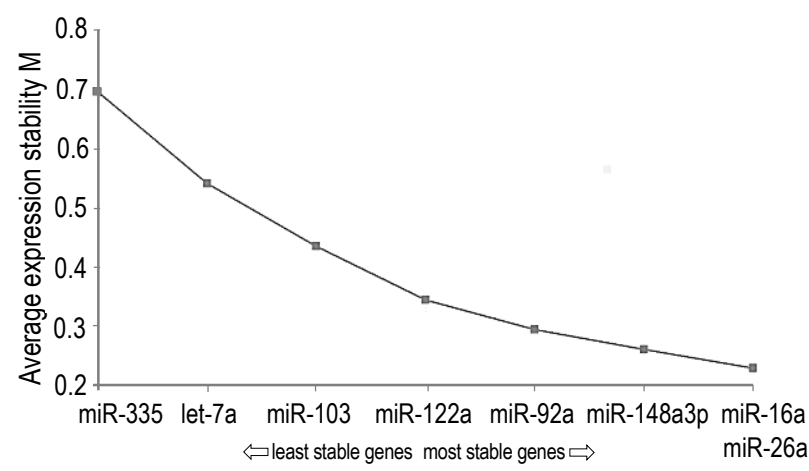

Figure 1. Stability of miRNA by GeNorm software analysis

qPCR analysis of miR-122a, miR-103, let-7a, miR-92a and miR-335 expression differences across the diet groups

According to the results of the GeNorm analysis, the normalized relative quantity of five
miRNAs (miR-122a, miR-103, let-7a, miR-92a and miR-335) using miR-16a, miR-26a and miR-148a-3p as endogenous controls was calculated. Statistical analysis with diet and sex as fixed factors showed that expression was not affected by sex in any of the miRNAs. Only miR-122a expression was different across the diet groups $(P<0.03)$. miR-122a expression was significantly lower in the liver of pigs fed diet with DDGS and coconut oil than in pigs fed diet with DDGS and beef tallow $(P<0.027)$, the group fed diet without DDGS but with the addition of rapeseed oil (-DDGS+rapeseed oil) $(P<0.0052)$ and the group fed DDGS and rapeseed oil in the $\operatorname{diet}(P<0.035)$.

The differences in miR-122a expression were relatively low (fold change 1.5-1.4) but statistically significant. No significant change in the expression of the other analysed miRNAs across the diet treatments was found; nevertheless, some trends were observed for miR-335 $(P<0.096)$. The mean expression of miR-335 was 2-fold lower in the -DDGS + rapeseed oil group than in the other groups (Figure 2); however, it was weakly expressed in the liver, and a high standard deviation was observed within the groups.

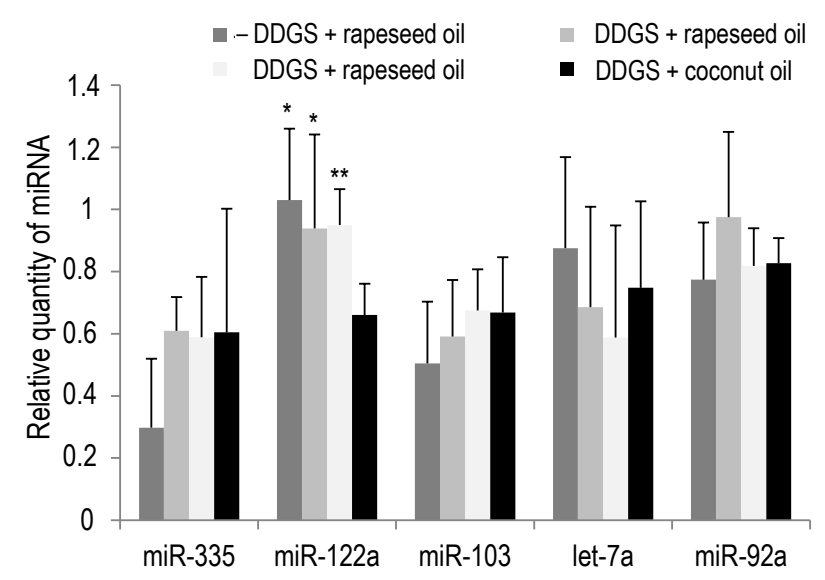

Figure 2. Relative quantity of selected miRNA in the liver of pigs fed four experimental diets with or without maize dried distilled grains with solubles (DDGS) addition, and with 3 different dietary fats: rapeseed oil, coconut oil and beef tallow after normalization to miR-16a, miR-26a and miR-148a-3p; * and ** indicate values statistically different from DDGS+coconut oil group at $P<0.05$ or $P<0.01$, respectively

\section{Discussion}

The obtained results on the stability of miRNA expression are in partial agreement with those of Timoneda et al. (2012) who investigated the stability of ten miRNAs in various tissues and breeds of pig. It was observed that all investigated miRNAs were stable and the expression of selected miRNAs was 
primarily influenced by the tissue, while the breed was not a determining factor. Three out of the ten analysed miRNAs (miR-26a, miR-103 and let-7a) were recommended for qPCR experiments in the liver samples; however, miR-148a-3p and miR-122a were not investigated in that study. The results of the present study suggest that other miRNAs may be better endogenous controls (miR-16a and miR-148a-3p) under the applied experimental conditions.

The results obtained in the present study also show that the addition of coconut oil to the diet with maize DDGS changes miR-122a expression in pig livers. miR-122a is one of the most abundant miRNAs in mammalian liver. miR-122a was extensively studied after the discovery that it may modulate Hepatitis C Virus (HCV) mRNA expression, making it a potential target for antiviral intervention (Jopling et al., 2005). It has been shown that miR-122a is engaged in many liver diseases, like cancer, hepatitis, steatosis and cirrhosis (Bandiera et al., 2015). Furthermore, miR-122a is associated with various metabolic processes, including cholesterol metabolism. It has been shown that miR-122a inhibition results in decreased cholesterol levels in mice (Esau et al., 2006) and in African green monkeys (Elmén et al., 2008) as well as decreased mRNA level of two key genes (HMGCS1 and HMGCR) involved in cholesterol biosynthesis (Krützfeldt et al., 2005). Interestingly, it was previously shown that the level of miR-122a expression depends on nutrition factors. Maternal high fat diet (HFD) alters the level of miR-122a expression in the liver of mouse offspring (Benatti et al., 2014). Lower expression of miR-122a was observed in the offspring of mothers fed HFD when compared with the offspring of mothers fed a standard chow diet during pregnancy and lactation (Benatti et al., 2014). Furthermore, miR-122a was down-regulated in the adipose tissue of mice after a long-term HFD feeding (Chartoumpekis et al., 2012).

On the other hand, in some experiments it has been shown that virgin coconut oil has hepato-protective activity on rabbits (Zakaria et al., 2011). Moreover, it was shown that the replacement of soyabean oil by coconut oil in the feed of broilers lowered the level of low density lipoprotein (LDL)-cholesterol and favourably affected lipid profile in serum (Wang et al., 2015). It is plausible that this effect may be related to the altered expression of miR-122a.

\section{Conclusions}

MicroRNAs play an important role in the regulation of gene expression. Nutrigenomic research should investigate this type of gene regulation because it may help to evaluate the molecular mechanism by which nutrients influence gene expression. Our experiment indicates that three miRNAs - miR16a, miR-26a and miR-148a-3p - are very stable in the liver of pigs fed different diets. On the other hand, miR-122a expression was altered in the liver of animals fed diet based on maize dried distilled grains with solubles (DDGS) with coconut oil. This finding sheds new light on nutrient-gene interactions and provides a basis for further research on the influence of nutrients on gene expression.

\section{Acknowledgement}

This work was financed by the National Science Centre Poland, Grant No. 2014/13/B/NZ9/02134.

\section{References}

Bandiera S., Pfeffer S., Baumert T.F., Zeisel M.B., 2015. miR-122 a key factor and therapeutic target in liver disease. J. Hepatol. 62, 448-457, https://doi.org/10.1016/j.jhep.2014.10.004

Benatti R.O., Melo A.M., Borges F.O., Ignacio-Souza L.M., Simino L.A.P., Milanski M., Velloso L.A., Torsoni M.A., Torsoni A.S., 2014. Maternal high-fat diet consumption modulates hepatic lipid metabolism and microRNA-122 (miR-122) and microRNA-370 (miR-370) expression in offspring. Br. J. Nutr. 111, 2112-1122, https://doi.org/10.1017/S0007114514000579

Chartoumpekis D.V., Zaravinos A., Ziros P.G., Iskrenova R.P., Psyrogiannis A.I., Kyriazopoulou V.E., Habeos I.G., 2012. Differential expression of microRNAs in adipose tissue after long-term high-fat diet-induced obesity in mice. PLOS ONE 7, e34872, https://doi.org/10.1371/journal.pone.0034872

Elmén J., Lindow M., Schütz S. et al., 2008. LNA-mediated microRNA silencing in non-human primates. Nature 452, 896-899, https:// doi.org/10.1038/nature06783

Esau C., Davis S., Murray S.F. et al., 2006. miR-122 regulation of lipid metabolism revealed by in vivo antisense targeting. Cell Metab. 3, 87-98, https://doi.org/10.1016/j.cmet.2006.01.005

Gamazon E.R., Innocenti F., Wei R. et al., 2013. A genome-wide integrative study of microRNAs in human liver. BMC Genomics 14, 395, https://doi.org/10.1186/1471-2164-14-395

Griffiths-Jones S., Grocock R.J., van Dongen S., Bateman A., Enright A.J., 2006. miRBase: microRNA sequences, targets and gene nomenclature. Nucleic Acids Res. 34, D140-D144, https://doi.org/10.1093/nar/gkj112

Griffiths-Jones S., Saini H.K., van Dongen S., Enright A.J., 2008. miRBase: tools for microRNA genomics. Nucleic Acids Res. 36, D154-D158, https://doi.org/10.1093/nar/gkm952

Jiménez-Chillarón J.C., Díaz R., Martínez D., Pentinat T., RamónKrauel M., Ribó S., Plösch T., 2012. The role of nutrition on epigenetic modifications and their implications on health. Biochimie 94, 2242-2263, https://doi.org/10.1016/j.biochi.2012.06.012

Jopling C.L., Yi M., Lancaster A.M., Lemon S.M., Sarnow P., 2005. Modulation of hepatitis $C$ virus RNA abundance by a liverspecific microRNA. Science 309, 1577-1581, https://doi. org/10.1126/science. 1113329 
Krützfeldt J., Rajewsky N., Braich R., Rajeev K.G., Tuschl T., Manoharan M., Stoffel M., 2005. Silencing of microRNAs in vivo with 'antagomirs'. Nature 438, 685-689, https://doi.org/10.1038/ nature 04303

Lee Y., Ahn C., Han J. et al., 2003. The nuclear RNase III Drosha initiates microRNA processing. Nature 425, 415-419, https://doi. org/10.1038/nature01957

Moore K.J., Rayner K.J., Suárez Y., Fernández-Hernando C., 2010. microRNAs and cholesterol metabolism. Trends Endocrinol. Metab. 21, 699-706, https://doi.org/10.1016/j. tem.2010.08.008

Müller M., Kersten S., 2003. Nutrigenomics: goals and strategies. Nat. Rev. Genet. 4, 315-322, https://doi.org/10.1038/nrg1047

Oczkowicz M., Świątkiewicz M., Ropka-Molik K., Gurgul A., Żukowski K., 2016. Effects of different sources of fat in the diet of pigs on the liver transcriptome estimated by RNA-Seq. Ann. Anim. Sci. 16, 1073-1090, https://doi.org/10.1515/aoas2016-0033

Palmer J.D., Soule B.P., Simone B.A., Zaorsky N.G., Jin L., Simone N.L., 2014. MicroRNA expression altered by diet: Can food be medicinal? Ageing Res. Rev. 17, 16-24, https://doi. org/10.1016/j.arr.2014.04.005

Pawlina K., Gurgul A., Oczkowicz M., Bugno-Poniewierska M., 2015. The characteristics of the porcine (Sus scrofa) liver miRNAome with the use of next generation sequencing. J. Appl. Genet. 56, 239-252, https://doi.org/10.1007/s13353-0140245-6

Pfaffl M.W., 2001. A new mathematical model for relative quantification in real-time RT-PCR. Nucleic Acids Res. 29, e45

Ross S.A., Davis C.D., 2011. MicroRNA, nutrition, and cancer prevention. Adv. Nutr. 2, 472-485, https://doi.org/10.3945/ an.111.001206

Saini S., Majid S., Dahiya R., 2010. Diet, microRNAs and prostate cancer. Pharm. Res. 27, 1014-1026, https://doi.org/10.1007/ s11095-010-0086-x

Sartor R.B., 2005. Probiotic therapy of intestinal inflammation and infections. Curr. Opin. Gastroenterol. 21, 44-50
Świątkiewicz M., Oczkowicz M., Ropka-Molik K., Hanczakowska E., 2016. The effect of dietary fatty acids composition on adipose tissue quality and expression of genes related to lipid metabolism in porcine livers. Anim. Feed Sci. Technol. 216, 204-215, https://doi.org/10.1016/j.anifeedsci.2016.03.020

Tavazoie S.F., Alarcon C., Oskarsson T., Padua D., Wang Q., Bos P.D., Gerald W.L., Massagué J., 2008. Endogenous human microRNAs that suppress breast cancer metastasis. Nature 451, 147-152, https://doi.org/10.1038/nature06487

Thomson D.W., Bracken C.P., Goodall G.J., 2011. Experimental strategies for microRNA target identification. Nucleic Acids Res. 39, 6845-6853, https://doi.org/10.1093/nar/gkr330

Timoneda O., Balcells I., Córdoba S., Castelló A., Sánchez A., 2012. Determination of reference microRNAs for relative quantification in porcine tissues. PLoS ONE 7, e44413, https://doi. org/10.1371/journal.pone.0044413

Vandesompele J., De Preter K., Pattyn F., Poppe B., Van Roy N., De Paepe A., Speleman F., 2002. Accurate normalization of real-time quantitative RT-PCR data by geometric averaging of multiple internal control genes. Genome Biol. 3, research0034.1-research0034.11

Vasudevan S., Tong Y., Steitz J.A., 2007. Switching from repression to activation: microRNAs can up-regulate translation. Science 318, 1931-1934, https://doi.org/10.1126/science.1149460

Wang J., Wang X., Li J., Chen Y., Yang W., Zhang L., 2015. Effects of dietary coconut oil as a medium-chain fatty acid source on performance, carcass composition and serum lipids in male broilers. Asian Australas. J. Anim Sci. 28, 223-230, https:// doi.org/10.5713/ajas. 14.0328

Zakaria Z.A., Rofiee M.S., Somchit M.N., Zuraini A., Sulaiman M.R., The L.K., Salleh M.Z., Long K., 2011. Hepatoprotective activity of dried- and fermented-processed virgin coconut oil. Evid.-based Complement. Altern. Med. 2011, 142739, https:// doi.org/10.1155/2011/142739 